\title{
ON WEIGHTED POLYNOMIAL APPROXIMATION WITH GAPS
}

\author{
GUANTIE DENG
}

\begin{abstract}
Let $\alpha$ be a nonnegative continuous function on $\mathbb{R}$. In this paper, the author obtains a necessary and sufficient condition for polynomials with gaps to be dense in $C_{\alpha}$, where $C_{\alpha}$ is the weighted Banach space of complex continuous functions $f$ on $\mathbb{R}$ with $f(t) \exp (-\alpha(t))$ vanishing at infinity.
\end{abstract}

\section{$\S 1$. Introduction}

Let $\alpha(t)$ be a nonnegative continuous function on $\mathbb{R}$, which is, henceforth, called a weight, defined on $\mathbb{R}$. We usually suppose that

$$
\lim _{|t| \rightarrow \infty} \frac{\alpha(t)}{\log |t|}=\infty
$$

Given a weight $\alpha(t)$, we consider the weighted Banach space $C_{\alpha}$ consisting of complex continuous functions $f(t)$ on $\mathbb{R}$ with $f(t) \exp (-\alpha(t))$ vanishing at infinity, and define

$$
\|f\|_{\alpha}=\sup \left\{\left|f(t) e^{-\alpha(t)}\right|: t \in \mathbb{R}\right\}
$$

for $f \in C_{\alpha}$. The classical Bernstein problem on weighted polynomial approximation is as follows: determine whether or not the polynomials are dense in the space $C_{\alpha}$ in the norm \|\|$_{\alpha}$; see [2], [3]. In this direction we mention one result, for example, the sufficiency of the above problem was obtained by S. Izumi and T. Kawata in 1937 in [7]. Later on, several authors obtained this result in different forms (for example, T. Hall ([6]), de Branges ([5]) and A. Borichev ([2], [3])).

Received October 21, 2003.

Revised March 8, 2004.

2000 Mathematics Subject Classification: 30B60, 41A30.

The project was supported by NSFC (Grant No. 10071005 and 10371011) and by SRF for ROCS.SEM. 
Theorem A. ([2], [3], [5], [6], [7]) Suppose that $\alpha(t)$ is an even function satisfying (1) and $\alpha\left(e^{t}\right)$ is a convex function on $\mathbb{R}$. Then a necessary and sufficient condition for polynomials to be dense in the space $C_{\alpha}$ is

$$
\int_{-\infty}^{+\infty} \frac{\alpha(t)}{1+t^{2}} d t=\infty
$$

The Müntz Theorem ([4]) naturally leads us to consider the density of polynomials with gaps in the space $C_{\alpha}$. Denote by $M(\Lambda)$ the space of polynomials with gaps which are finite linear combinations of the system $\left\{t^{\lambda}: \lambda \in \Lambda\right\}$, where $\Lambda=\left\{\lambda_{n}: n \in \mathbb{N}\right\}$ is a sequence of strictly increasing positive integers. The condition (1) guarantees that $M(\Lambda)$ is a subspace of $C_{\alpha}$; we then ask whether $M(\Lambda)$ is dense in $C_{\alpha}$ in the norm \|\|$_{\alpha}$ - this is the so-called weighted polynomial approximation with gaps, which is similar to the classical Bernstein problem on weighted polynomial approximation. Motivated by Bernstein's problem and Malliavin's Method ([8]), we find a necessary and sufficient condition for $M(\Lambda)$ to be dense in $C_{\alpha}$. The main result is as follows.

THEOREM. Suppose that $\alpha(t)$ is an even function satisfying (1) and $\alpha\left(e^{t}\right)$ is convex function on $\mathbb{R}$. Let $\Lambda=\left\{\lambda_{n}: n \in \mathbb{R}\right\}$ is a sequence of strictly increasing positive integers and let

$$
\Lambda(r)= \begin{cases}2 \sum_{\lambda_{n} \leq r} \frac{1}{\lambda_{n}}, & \text { if } r \geq \lambda_{1} \\ 0, & \text { otherwise }\end{cases}
$$

$k(r)=\Lambda(r)-\log ^{+} r, \log ^{+} r=\max \{\log r, 0\}, \widetilde{k}(r)=\inf \left\{k\left(r^{\prime}\right): r^{\prime} \geq r\right\}$.

If

$$
\int_{0}^{+\infty} \frac{\alpha(\exp \{\widetilde{k}(t)-a\})}{1+t^{2}} d t=\infty
$$

for each $a \in \mathbb{R}$, then $M(\Lambda)$ is dense in $C_{\alpha}$.

Conversely, if the sequence $\Lambda$ contains all of the positive odd integers, then for $M(\Lambda)$ to be dense in $C_{\alpha}$, it is necessary that (4) holds for each $a \in \mathbb{R}$.

Remark. Since $\sum_{n \leq r} \frac{1}{n}-\log r$ converges to Euler's constant $\gamma$, as $r \rightarrow$ $\infty$, the condition (4) is equivalent to the condition (2) in the case that $\Lambda=$ $\mathbb{N}=\{1,2, \ldots\}$. Therefore our theorem is a generalization of Theorem A. If 
$\Lambda$ contains all of the positive odd integers $2 \mathbb{N}-1=\{2 k-1: k=1,2, \ldots\}$, then $\widetilde{k}(r)=\widetilde{\Lambda}(r)+O(1)(r \rightarrow \infty)$, where $\widetilde{\Lambda}(r)$ is defined by (3) with $\Lambda$ replaced by $\widetilde{\Lambda}=\{\lambda+1: \lambda \in \Lambda, \lambda$ even $\}$. In this case, $\widetilde{k}(r)$ in the integral of (4) can be replaced by $\widetilde{\Lambda}(r)$. Moreover, we conjecture that the condition (4) is also necessary for polynomials with gaps to be dense in the space $C_{\alpha}$, if we remove the the restriction that $\Lambda$ contains all of the positive odd integers.

\section{§2. Proof of Theorem}

In order to prove our theorem, we need some technical lemmas (Hereafter we denote a positive constant by $A$, not necessarily the same at each occurrence).

LEMMA 1. ([8]) Let $\beta(t)$ be a nonnegative convex function on $\mathbb{R}$ such that $\beta(\log |t|)$ satisfies $(1)$, and assume that

$$
\beta^{*}(t)=\sup \{x t-\beta(x): x \in \mathbb{R}\}, \quad t \in \mathbb{R}
$$

is the Young transform of the function $\beta(x)$; see [9]. Let $\widetilde{k}(r)$ be a increasing function on $[0, \infty)$ and there exist a positive constant $A$ such that

$$
\widetilde{k}(R)-\widetilde{k}(r) \leq A(\log R-\log r+1)
$$

for $R>r>1$. Let $f(z)$ be an analytic function in $\mathbb{C}_{+}$and there exist a positive constant $A$ such that

$$
|f(z)| \leq A \exp \{A x+\beta(x)-x \widetilde{k}(|z|)\}, \quad z=x+i y \in \mathbb{C}_{+} .
$$

If

$$
\int_{1}^{+\infty} \frac{\beta^{*}(\widetilde{k}(t)-a)}{1+t^{2}} d t=\infty
$$

for each real number a, then $f(z) \equiv 0$.

LEMMA 2. ([1]) If $\Lambda$ is a sequence of increasing positive integrals, then the function

$$
G_{\Lambda}(z)=\prod_{n=1}^{\infty}\left(\frac{\lambda_{n}-z}{\lambda_{n}+z}\right) \exp \left(\frac{2 z}{\lambda_{n}}\right)
$$


is analytic in the closed right half plane $\overline{\mathbb{C}}_{+}=\{z=x+i y: x \geq 0\}$, and there exists a positive constant $A$ such that

$$
|\log | G_{\Lambda}(z)|-x \Lambda(|z|)| \leq A x, \quad z=x+i y \in D,
$$

where $\Lambda(r)$ is defined by (3) and $D=\left\{z \in \overline{\mathbb{C}}_{+}:\left|z-\lambda_{n}\right| \geq \frac{1}{8}, n \in \mathbb{R}\right\}$.

Proof of Theorem. By the Hahn-Banach theorem, we need to prove that if $T$ is a bounded linear functional on $C_{\alpha}$ and $T\left(t^{\lambda}\right)=0$ for $\lambda \in \Lambda$, then $T=0$. So let $T$ be a bounded linear functional on $C_{\alpha}$ and $T\left(t^{\lambda}\right)=0$ for $\lambda \in \Lambda$; then by the Riesz representation theorem, there exists a complex measure $\mu$ such that

$$
\int_{-\infty}^{+\infty} e^{\alpha(t)} d|\mu|(t)=\|T\|
$$

and

$$
T(h)=\int_{-\infty}^{+\infty} h(t) d \mu(t)
$$

for $h \in C_{\alpha}$. Therefore the function

$$
f_{0}(z)=e^{\frac{\pi}{2} i z} \int_{0}^{+\infty} t^{z} d \mu(t)+e^{-\frac{\pi}{2} i z} \int_{-\infty}^{0}|t|^{z} d \mu(t)
$$

is analytic in the open right half-plane $\mathbb{C}_{+}$, continuous in the closed right half-plane $\overline{\mathbb{C}}_{+}=\{z=x+i y: x \geq 0\}, f_{0}(\lambda)=0, \lambda \in \Lambda$ and

$$
\left|f_{0}(z)\right| \leq\|T\| \exp \left\{\beta(x)+\frac{\pi}{2}|y|\right\}
$$

for $z=x+i y \in \mathbb{C}_{+}$, where

$$
\beta(x)=\sup \{x \log t-\alpha(t): t>0\}
$$

is the Young transform of the convex function $\alpha\left(e^{s}\right)$. Let $G_{\Lambda}(z)$ be defined by $(9)$ and $\Gamma(z)$ be The Gamma function. By (10) and the Stirling asymptotic formula, we see that there exists a positive constant $A$ such that the function

$$
f(z)=\frac{f_{0}(z)}{G(z) \Gamma(1+z)}
$$

satisfies

$$
|f(z)| \leq A \exp \{\beta(x)-x \widetilde{k}(|z|)+A x\}
$$


where $\widetilde{k}(r)=\inf \left\{\Lambda\left(r^{\prime}\right)-\log ^{+} r^{\prime}: r^{\prime} \geq r\right\}$ satisfies (6) with $A=1$. We may assume, without loss of generality, that $\alpha(1)=0$. As is known, $\beta(x)$ is a convex nonnegative function which also satisfies $\beta(0)=0$ and

$$
\sup \{x s-\beta(x): x \geq 0\}=\alpha\left(e^{s}\right) .
$$

We see from Lemma 1 and $(4)$ that $f(z) \equiv 0$, so $f_{0}(z) \equiv 0$. In particular $f_{0}(n)=0, n=0,1,2, \ldots$ Therefore $T\left(t^{n}\right)=0, n=0,1,2, \ldots$. Since the condition (3) implies the condition (2), $T=0$ by Theorem A. This completes the proof of the necessity of the theorem.

Conversely, assume that the sequence $\Lambda$ contains all of the odd positive integers $2 \mathbb{N}-1$, then $\widetilde{k}(r)=\widetilde{\Lambda}(r)+O(1)(r \rightarrow \infty)$, where $\widetilde{\Lambda}(r)$ is defined by (3) with $\Lambda$ replaced by $\widetilde{\Lambda}=\{\lambda+1: \lambda \in \Lambda, \lambda$ even $\}$. In this case, $\widetilde{k}(r)$ in the integral of (4) can be replaced by $\widetilde{\Lambda}(r)$. $\widetilde{k}(r)=k(r)+O(1)(r \rightarrow \infty)$. Assume that there exists a real number $a$ such that the integral

$$
\int_{0}^{\infty} \frac{\alpha(\exp \{\widetilde{\Lambda}(t)-a\})}{1+t^{2}} d t<\infty
$$

Let $\varphi(t)$ be an even function such that $\varphi(t)=\alpha(\exp \{\widetilde{\Lambda}(t)-a\})$ for $t \geq 0$ and let $u(z)$ be the the Poisson integral of $\varphi(t)$, i.e.,

$$
u(x+i y)=\frac{x}{\pi} \int_{-\infty}^{+\infty} \frac{\varphi(t)}{x^{2}+(y-t)^{2}} d t .
$$

Then $u(x+i y)$ is harmonic in the half-plane $\mathbb{C}_{+}$and there exists an analytic function $g_{1}(z)$ on $\mathbb{C}_{+}$satisfying

$$
\begin{aligned}
\operatorname{Re} g_{1}(z) & =u(z) \geq \frac{4 x}{\pi} \int_{|t| \geq|z|} \frac{\varphi(t)}{x^{2}+(y-t)^{2}} d t \\
& \geq \varphi(|z|)=\alpha(\exp \{\widetilde{\Lambda}(|z|)-a\}) \\
& \geq(x-1)(\widetilde{\Lambda}(|z|)-a)-\beta(x-1)
\end{aligned}
$$

where $z=x+i y, r=|z|, x>1$. Let

$$
g_{0}(z)=\frac{G_{\widetilde{\Lambda}}(z)}{(1+z)^{N}} \exp \left\{-g_{1}(z)-N z-N\right\},
$$

where $N$ is a large positive integer and $G_{\widetilde{\Lambda}}(z)$ is defined by (9). By (9) and (10), we have $g_{0}(\lambda+1)=0$ for $\lambda \in \Lambda, \lambda$ even and

$$
\left|g_{0}(z)\right| \leq \frac{1}{1+|z|^{2}} \exp \{\beta(x-1)-x\}, \quad z \in \mathbb{C}_{+} .
$$


Let

$$
h_{0}(t)=\frac{1}{\sqrt{2 \pi}} \int_{-\infty}^{+\infty} g_{0}\left(\frac{1}{2}+i y\right) t^{-\left(\frac{1}{2}+i y\right)} d y .
$$

Then $h_{0}(t)$ is continuous on $[0,+\infty)$. By the Cauchy formula,

$$
h_{0}(t)=\frac{1}{\sqrt{2 \pi}} \int_{-\infty}^{+\infty} g_{0}(x+i y) t^{-(x+i y)} d y
$$

for $x>0$. We obtain from (11), (12) and (13) that

$$
\left|h_{0}(t)\right| \leq \exp (-\alpha(t)-|\log t|)
$$

and

$$
g_{0}(z)=\frac{1}{\sqrt{2 \pi}} \int_{0}^{+\infty} h_{0}(t) t^{z-1} d t
$$

for $x>0$. We extend the function $h_{0}(t)$ to an even function by letting $h_{0}(t)=h_{0}(-t)$ for $t<0$. Therefore the bounded linear functional

$$
T(h)=\frac{1}{\sqrt{2 \pi}} \int_{-\infty}^{+\infty} h_{0}(t) h(t) d t \quad\left(h \in C_{\alpha}\right)
$$

satisfies $T\left(t^{\lambda}\right)=0$ for $\lambda \in \Lambda$, and

$$
\|T\|=\frac{2}{\sqrt{2 \pi}} \int_{0}^{+\infty}\left|h_{0}(t)\right| e^{\alpha(t)} d t>0 .
$$

By the Riesz representation theorem, the space $M(\Lambda)$ is not dense in $C_{\alpha}$. This completes the proof of the theorem.

Acknowledgements. The author would like to express his deep gratitude to the referee for his valuable comments and suggestions.

\section{REFERENCES}

[1] R. P. Boas, Jr., Entire Functions, Academic Press, New York, 1954.

[2] A. Borichev, On weighted polynomial approximation with monotone weights, Proc. Amer. Math. Soc., 128 (2000), no. 12, 3613-3619.

[3] A. Borichev, On the closure of polynomials in weighted space of functions on the real line, Indiana Univ. Math. J., 50 (2001), no. 2, 829-845.

[4] P. B. Borwein and T. Erdélyi, Polynomials and Polynomial Inequalities, SpringerVerlag, New York, N. Y., 1995. 
[5] L. de Branges, The Bernstein problem, Proc. Amer. Math. Soc., 10 (1959), 825-832.

[6] T. Hall, Sur l'approximation polynômiale des fonctions continues d'une variable réelle, Neuvième Congrès des Mathémticiens Scandianaves (1938), Helsingfors (1939), pp. 367-369.

[7] S. Izumi and T. Kawata, Quasi-analytic class and closure of $\left\{t^{n}\right\}$ in the interval $(-\infty, \infty)$, Tôhoku Math. J., 43 (1937), 267-273.

[8] P. Malliavin, Sur quelques procédés d'extrapolation, Acta Math., 83 (1955), 179-255.

[9] R. Rockafellar, Convex analysis, Princeton Univ. Press, Princeton, 1970.

Department of Mathematics

Beijing Normal University

100875 Beijing

The People's Republic of China

denggt@bnu.edu.cn 